\title{
A weak type bound for a singular integral
}

\section{Andreas Seeger}

Abstract. A weak type $(1,1)$ estimate is established for the first order $d$-commutator introduced by Christ and Journé, in dimension $d \geq 2$.

\section{Introduction}

Let $K$ be regular Calderón-Zygmund convolution kernel on $\mathbb{R}^{d}, d \geq 2$, i.e. $K \in \mathcal{S}^{\prime}$, is locally bounded in $\mathbb{R}^{d} \backslash\{0\}$ and satisfies

$$
|K(x)| \leq A|x|^{-d} \quad x \neq 0,
$$

and, for some $\varepsilon \in(0,1]$,

$$
|K(x+h)-K(x)| \leq A|h|^{\varepsilon}|x|^{-d-\varepsilon} \quad \text { if }|x|>2|h| ;
$$

moreover

$$
\|\widehat{K}\|_{\infty} \leq A<\infty .
$$

Let $a \in L^{\infty}\left(\mathbb{R}^{d}\right)$. The so-called $d$-commutator $T \equiv T[a]$ of first order associated with $K$ and $a$ is defined for Schwartz functions $f$ by

$$
T[a] f(x)=p . v . \int K(x-y) \int_{0}^{1} a(s x+(1-s) y) d s f(y) d y .
$$

In dimensions $d \geq 2$ this definition yields a rough analog of the Calderón commutator [1] in one dimension. Christ and Journé [3] proved that $T$ and higher order versions extend to bounded operators on $L^{p}\left(\mathbb{R}^{d}\right)$, for $1<p<\infty$. We prove that the first order $d$-commutator is also of weak type $(1,1)$.

Theorem 1.1. There is $C_{d}<\infty$ so that for any $f \in L^{1}\left(\mathbb{R}^{d}\right)$ and any $a \in L^{\infty}\left(\mathbb{R}^{d}\right)$,

$$
\sup _{\lambda>0} \lambda \operatorname{meas}\left(\left\{x \in \mathbb{R}^{d}:|T[a] f(x)|>\lambda\right\}\right) \leq C_{d} A \frac{1}{\varepsilon} \log \left(\frac{2}{\varepsilon}\right)\|a\|_{\infty}\|f\|_{L^{1}\left(\mathbb{R}^{d}\right)} .
$$

Mathematics Subject Classification (2010): 42B15.

Keywords: Rough singular integrals, $d$-commutators, Christ-Journé operators, weak type $(1,1)$ bounds. 
In dimension two this result has recently been established by Grafakos and Honzík [6] (assuming $\varepsilon=1$ ). Their approach relies on a method developed in [2], [4] and [7] for proving a weak type $(1,1)$ bound for rough singular convolution operators. A dyadic decomposition $T[a]=\sum T_{j}$ is used on the kernel side, and the argument relies on the fact that in dimension two the kernels of the operators $T_{j}^{*} T_{i}$ have certain Hölder continuity properties. This argument is no longer valid for higher dimension. It is conceivable that for $d \geq 3$ one might be able to develop the more complicated iterated $T^{*} T$ arguments introduced by Christ and Rubio de Francia [4] and further extended by Tao [11], but this route would lead to substantial technical difficulties and we shall not pursue it. Our approach is different and relies on an idea introduced in [8]. An orthogonality argument for a microlocal decomposition of the operator is used. The implementation of this idea in the present setting is more complicated in the convolution case as the Christ-Journé operators can be viewed as an amalgam of operators of generalized convolution type (for which there is a suitable calculus of wavefront sets) and operators of multiplication with a rough function.

Notation. We write $\mathcal{E}_{1} \lesssim \mathcal{E}_{2}$ to indicate that $\mathcal{E}_{1} \leq C \mathcal{E}_{2}$ for some 'constant' $C$ that may depend on $d$. We also use the notation $\lesssim_{N}$ to indicate dependence on other parameters $N$. We denote by $\widehat{f}$ or $\mathcal{F} f$ the Fourier transform of $f$, defined for Schwartz functions by $\widehat{f}(\xi)=\int f(y) e^{-i\langle y, \xi\rangle} d y$.

This paper. In $\S 2$ we outline the proof of Theorem 1.1 with the three technical propositions $2.2,2.3,2.4$ proved in $\S 3, \S 4, \S 5$, respectively. In $\S 6$ we shall mention some open problems.

\section{Decompositions and auxiliary estimates}

We may assume that $A \leq 1,\|a\|_{\infty} \leq 1$ and write $T=T[a]$. Fix $f \in L^{1}\left(\mathbb{R}^{d}\right)$. We use the standard Calderón-Zygmund decomposition of $f$ at height $\lambda$ (see [10]). Then

$$
f=g+b=g+\sum_{Q \in \mathfrak{Q}_{\lambda}} b_{Q}
$$

where $\|g\|_{\infty} \leq \lambda,\|g\|_{1} \lesssim\|f\|_{1}$, each $b_{Q}$ is supported in a dyadic cube $Q$ with sidelength $2^{L(Q)}$ and center $y_{Q}$, and $\mathfrak{Q}_{\lambda}$ is a family of dyadic cubes with disjoint interiors. Moreover $\left\|b_{Q}\right\|_{1} \lesssim \lambda|Q|$ for each $Q \in \mathfrak{Q}_{\lambda}$ and $\sum_{Q \in \mathfrak{Q}_{\lambda}}|Q| \lesssim \lambda^{-1}\|f\|_{1}$. For each $Q$ let $Q^{*}$ be the dilate of $Q$ with same center and $L\left(Q^{*}\right)=L(Q)+10$, and let $E=\bigcup_{Q \in \mathfrak{Q}_{\lambda}} Q^{*}$. Then also

$$
\operatorname{meas}(E) \lesssim \lambda^{-1}\|f\|_{1}
$$

Finally, for each $Q$, the mean value of $b_{Q}$ vanishes:

$$
\int b_{Q}(y) d y=0
$$


Since $T$ is bounded on $L^{2}$ (cf. [3]) we have, as in standard Calderón-Zygmund theory,

$$
\|T g\|_{2}^{2} \leq\|T\|_{L^{2} \rightarrow L^{2}}^{2}\|g\|_{2}^{2} \lesssim\|g\|_{1}\|g\|_{\infty} \lesssim \lambda\|g\|_{1}
$$

the estimate for the good function $g$. By Chebyshev's inequality,

$$
\left|\left\{x \in \mathbb{R}^{d}:|T g(x)|>\lambda / 10\right\}\right| \leq 100 \lambda^{-2}\|T g\|_{2}^{2} \lesssim \lambda^{-1}\|g\|_{1} \lesssim \lambda^{-1}\|f\|_{1} .
$$

We use a dyadic decomposition of the kernel. Let $\varphi$ be a radial $C^{\infty}$ function, so that $\varphi(x)=1$ for $|x| \leq 1$ and $\varphi(x)=0$ for $|x| \geq 6 / 5$. Let

$$
K_{j}(x)=\left(\varphi\left(2^{-j} x\right)-\varphi\left(2^{-j+1} x\right)\right) K(x)
$$

so that $K=\sum K_{j}$ in the sense of distributions on $\mathbb{R}^{d} \backslash\{0\}$ and $K_{j}$ is supported in the annulus $\left\{x: 2^{j-1} \leq|x| \leq \frac{6}{5} 2^{j}\right\}$. Let $T_{j}$ be the integral operator with Schwartz kernel

$$
K_{j}(x-y) \int_{0}^{1} a(s x+(1-s) y) d s .
$$

For $m \in \mathbb{Z}$ let

$$
B_{m}=\sum_{\substack{Q \in \mathfrak{Q}_{\lambda} \\ L(Q)=m}} b_{Q}
$$

Observe that for each $j$ and $m$ the function $T_{j} B_{m}$ belongs to $L^{1}$, and that

$$
\operatorname{supp}\left(T_{j} B_{m}\right) \subset E, \quad m \geq j .
$$

Moreover, for each $n$,

$$
\sum_{j}\left\|T_{j} B_{j-n}\right\|_{1} \lesssim\|f\|_{1}
$$

and thus, if

$$
n(\varepsilon)=10^{10} d \varepsilon^{-1} \log _{2}\left(2 \varepsilon^{-1}\right)
$$

we have by Chebyshev's inequality

$$
\begin{aligned}
& \operatorname{meas}\left(\left\{x \in \mathbb{R}^{d}: \sum_{0<n \leq n(\varepsilon)} \sum_{j}\left|T_{j} B_{j-n}(x)\right|>\lambda / 10\right\}\right) \\
& \lesssim \varepsilon^{-1} \log \left(2 \varepsilon^{-1}\right) \lambda^{-1}\|f\|_{1} .
\end{aligned}
$$

It thus suffices to show that $\sum_{n>n(\varepsilon)}\left(\sum_{j} T_{j} B_{j-n}\right)$ converges in the topology of $\left(L^{1}+L^{2}\right)\left(\mathbb{R}^{d} \backslash E\right)$ and satisfies the inequality

$$
\operatorname{meas}\left(\left\{x \in \mathbb{R}^{d} \backslash E: \sum_{n>n(\varepsilon)}\left|\sum_{j} T_{j} B_{j-n}(x)\right|>4 \lambda / 5\right\}\right) \lesssim \lambda^{-1}\|f\|_{1} .
$$




\section{Finer decompositions}

We first slightly modify the kernel $K_{j}$ and subtract an acceptable error term which is small in $L^{1}$. In what follows assume $n>n(\varepsilon)$ as defined above. Let

$$
\ell(n)=\left[2 \log _{2}(n)\right]+2 \quad \text { and } \quad \ell_{\varepsilon}(n)=\left[2 \varepsilon^{-1} \log _{2} n\right]+2 .
$$

Let $\Phi$ be a radial $C_{0}^{\infty}$ function supported in $\{|x| \leq 1\}$, and satisfying $\int \Phi(x) d x=1$. Let $\Phi_{m}(x)=2^{-m d} \Phi\left(2^{-m} x\right)$. Define

$$
K_{j}^{n}=K_{j} * \Phi_{j-\ell_{\varepsilon}(n)}
$$

Then $K_{j}^{n}$ is supported in $\left\{x: 2^{j-2} \leq|x| \leq 2^{j+2}\right\}$, and, by the regularity assumption (1.2),

$$
\begin{aligned}
\left\|K_{j}-K_{j}^{n}\right\|_{1} & \lesssim 2^{-\left(j-\ell_{\varepsilon}(n)\right) d} \iint_{\substack{|h| \leq 2^{j-1-\ell_{\varepsilon}(n)} \\
2^{j-2} \leq|x| \leq 2^{j+2}}}\left|K_{j}(x)-K_{j}(x-h)\right| d x d h \\
& \lesssim 2^{-\ell_{\varepsilon}(n) \varepsilon} \lesssim n^{-2} .
\end{aligned}
$$

By differentiation and (1.1)

$$
\left|\partial^{\alpha} K_{j}^{n}(x)\right| \leq C_{\alpha} 2^{-j d} 2^{\left(\ell_{\varepsilon}(n)-j\right)|\alpha|} .
$$

Let $\vartheta_{n} \in C^{\infty}(\mathbb{R})$ be supported in $\left(n^{-2}, 1-n^{-2}\right)$, such that $\vartheta_{n}(s)=1$ for $s \in$ $\left[2 n^{-2}, 1-2 n^{-2}\right]$, and such that the derivatives of $\vartheta_{n}$ satisfy the natural estimates

$$
\left\|\vartheta_{n}^{(N)}\right\|_{\infty} \leq C_{N} n^{2 N}
$$

We then let $T_{j}^{n}$ be the integral operator with Schwartz kernel

$$
K_{j}^{n}(x-y) \int \vartheta_{n}(s) a(s x+(1-s) y) d s
$$

The following lemma is an immediate consequence of estimate (2.4) and the support property of $\vartheta_{n}$.

Lemma 2.1. The operator $T_{j}-T_{j}^{n}$ is bounded on $L^{1}$, with operator norm

$$
\left\|T_{j}-T_{j}^{n}\right\|_{L^{1} \rightarrow L^{1}} \lesssim n^{-2}
$$

Lemma 2.1 implies

$$
\begin{aligned}
\operatorname{meas}(\{x & \left.\left.: \sum_{n>n(\varepsilon)}\left|\sum_{j}\left(T_{j} B_{j-n}(x)-T_{j}^{n} B_{j-n}(x)\right)\right|>\lambda / 10\right\}\right) \\
& \leq 10 \lambda^{-1}\left\|\sum_{n>n(\varepsilon)} \sum_{j}\left|T_{j} B_{j-n}-T_{j}^{n} B_{j-n}\right|\right\|_{1} \\
& \lesssim \lambda^{-1} \sum_{n \geq 1} n^{-2} \sum_{j}\left\|B_{j-n}\right\|_{1} \lesssim \lambda^{-1}\|f\|_{1}
\end{aligned}
$$


and therefore it is enough to show

$$
\operatorname{meas}\left(\left\{x: \sum_{n>n(\varepsilon)} \sum_{j}\left|T_{j}^{n} B_{j-n}(x)\right|>\frac{7}{10} \lambda\right\}\right) \lesssim \lambda^{-1}\|f\|_{1} .
$$

For the proof of (2.7) we subtract various regular or small terms from the operators $T_{j}^{n}$. Let $\ell(n)$ be as in $(2.3)$ and denote by $P_{m}$ the convolution operator with convolution kernel $\Phi_{m}$ (defined following $(2.3)$ ). We have:

Proposition 2.2. For $n>1$,

$$
\left\|P_{j-n+\ell(n)} T_{j}^{n} B_{j-n}\right\|_{1} \lesssim n^{-2} \log n\left\|B_{j-n}\right\|_{1} .
$$

The proposition will be proved in $\S 3$. It yields

$$
\begin{aligned}
& \left.\operatorname{meas}\left(\left\{x \in \mathbb{R}^{d} \backslash E: \sum_{n>n(\varepsilon)} \mid \sum_{j} P_{j-n+\ell(n)} T_{j}^{n} B_{j-n}(x)\right) \mid>\lambda / 10\right\}\right) \\
& \lesssim 10 \lambda^{-1} \sum_{n>n(\varepsilon)} \sum_{j}\left\|P_{j-n+\ell(n)} T_{j}^{n} B_{j-n}\right\|_{1} \\
& \lesssim \lambda^{-1} \sum_{n>1} n^{-2} \log n \sum_{j}\left\|B_{j-n}\right\|_{1} \lesssim \lambda^{-1}\|f\|_{1}
\end{aligned}
$$

and thus we need to consider the term

$$
\sum_{n>n(\varepsilon)} \sum_{j}\left(I-P_{j-n+\ell(n)}\right) T_{j}^{n} B_{j-n}(x)
$$

and estimate the measure of the set where $|(2.8)|>3 \lambda / 5$. We will have to exploit the fact that the integral $\int_{0}^{1} a(s x+(1-s) y) d s$ smooths the rough function $a$ in the direction parallel to $x-y$, and use a microlocal decomposition which we now describe.

Let $1 / 10<\gamma<9 / 10$ (say $\gamma=1 / 2$ ), and let $\Theta_{n}$ be a set of unit vectors such that if $\nu \neq \nu^{\prime}, \nu, \nu^{\prime} \in \Theta_{n}$ then $\left|\nu-\nu^{\prime}\right| \geq 2^{-4-n \gamma}$, and assume that $\Theta_{n}$ is maximal with respect to this property. Note that

$$
\operatorname{card}\left(\Theta_{n}\right) \lesssim 2^{n \gamma(d-1)}
$$

For each $\nu$ we may choose a function $\tilde{\chi}_{n, \nu}$ on $C^{\infty}\left(S^{d-1}\right)$ with the property that $\tilde{\chi}_{n, \nu}(x) \geq 0, \tilde{\chi}_{n, \nu}(\theta)=1$ if $|\theta-\nu| \leq 2^{-3-n \gamma}, \tilde{\chi}_{n, \nu}(\theta)=0$ if $|\theta-\nu|>2^{-2-n \gamma}$, and such that for each $M \in \mathbb{N}$ the functions $2^{-n \gamma M} \tilde{\chi}_{n, \nu}$ form a bounded family in $C^{M}\left(S^{d-1}\right)$. For each $\theta$ there is at least one $\nu$ such that $\widetilde{\chi}_{n, \nu}(\theta)=1$, by the maximality assumption. Moreover, by the separatedness assumption the number of $\nu \in \Theta_{n}$ for which $\widetilde{\chi}_{n, \nu}(\theta) \neq 0$ is bounded above, uniformly in $\theta$ and $n$. Define, for $\nu \in \Theta_{n}$,

$$
\chi_{n, \nu}(x)=\frac{\widetilde{\chi}_{n, \nu}(x /|x|)}{\sum_{\nu^{\prime} \in \Theta_{n}} \widetilde{\chi}_{n, \nu^{\prime}}(x /|x|)} .
$$


Then $\sum_{\nu \in \Theta_{n}} \chi_{n, \nu}(x)=1$ for every $x \in \mathbb{R}^{d} \backslash\{0\}$ and by homogeneity we have the following estimates for multi-indices $\alpha$ and $x \neq 0$,

$$
\begin{aligned}
\left|(\langle\nu, \nabla\rangle)^{M} \chi_{n, \nu}(x)\right| & \leq C_{M}|x|^{-M}, \\
\left|\partial^{\alpha} \chi_{n, \nu}(x)\right| & \leq C_{\alpha} 2^{n \gamma|\alpha|}|x|^{-|\alpha|} .
\end{aligned}
$$

Let $K_{j}^{n, \nu}(x)=K_{j}^{n}(x) \chi_{n, \nu}(x)$ and let $T_{j}^{n, \nu}$ be the operator with Schwartz kernel

$$
K_{j}^{n, \nu}(x-y) \int \vartheta_{n}(s) a(s x+(1-s) y) d s .
$$

We then have

$$
T_{j}^{n}=\sum_{\nu \in \Theta_{n}} T_{j}^{n, \nu}
$$

Let $\phi \in C^{\infty}(\mathbb{R})$ so that $\phi(u)=1$ for $|u|<1 / 2$ and $\phi(u)=0$ for $|u| \geq 1$ and define the singular convolution operator $\mathfrak{S}_{n, \nu}$ by

$$
\widehat{\mathfrak{S}_{n, \nu} f}(\xi)=\phi\left(2^{n \gamma} n^{-5}\langle\nu, \xi /|\xi|\rangle\right) \widehat{f}(\xi) .
$$

The terms involving $\left(I-\mathfrak{S}_{n, \nu}\right) T_{j}^{n, \nu}$ can be dealt with by $L^{1}$ estimates. In $\S 4$ we shall prove:

Proposition 2.3. For $n>n(\varepsilon)$ and $\nu \in \Theta_{n}$,

$$
\left\|\sum_{j}\left(I-P_{j-n+\ell(n)}\right)\left(I-\mathfrak{S}_{n, \nu}\right) T_{j}^{n, \nu} B_{j-n}\right\|_{1} \lesssim n^{-2} 2^{-n \gamma(d-1)}\|f\|_{1} .
$$

For the rougher terms involving $\mathfrak{S}_{n, \nu} T_{j}^{n, \nu}$ we shall use a weak orthogonality argument from [8] to prove the following $L^{2}$ estimate.

Proposition 2.4. For $n>n(\varepsilon)$,

$$
\left\|\sum_{\nu \in \Theta_{n}} \sum_{j}\left(I-P_{j-n+\ell(n)}\right) \mathfrak{S}_{n, \nu} T_{j}^{n, \nu} B_{j-n}\right\|_{2}^{2} \lesssim 2^{-n \gamma} n^{5} \lambda\|f\|_{1} .
$$

Given these two propositions we can finish the outline of the proof of Theorem 1.1. Namely by Chebyshev's inequality,

$$
\begin{aligned}
\operatorname{meas} & \left(\left\{x:\left|\sum_{n>n(\varepsilon)} \sum_{j}\left(I-P_{j-n+\ell(n)}\right) T_{j}^{n} B_{j-n}(x)\right|>\frac{3}{5} \lambda\right\}\right) \\
& \lesssim 5 \lambda^{-1}\left\|\sum_{n>n(\varepsilon)} \sum_{\nu \in \Theta_{n}} \sum_{j}\left(I-P_{j-n+\ell(n)}\right)\left(I-\mathfrak{S}_{n, \nu}\right) T_{j}^{n, \nu} B_{j-n}\right\|_{1} \\
& +25 \lambda^{-2}\left\|\sum_{n>n(\varepsilon)} \sum_{\nu \in \Theta_{n}} \sum_{j}\left(I-P_{j-n+\ell(n)}\right) \mathfrak{S}_{n, \nu} T_{j}^{n, \nu} B_{j-n}\right\|_{2}^{2}
\end{aligned}
$$

and by Propositions 2.3 and 2.4 and Minkowski's inequality this is bounded by

$$
C \lambda^{-1}\|f\|_{1}\left(\sum_{n} n^{-2} 2^{-n \gamma(d-1)} \operatorname{card}\left(\Theta_{n}\right)+\sum_{n} 2^{-n \gamma} n^{5}\right) \lesssim \lambda^{-1}\|f\|_{1} .
$$




\section{Proof of Proposition 2.2}

Let $Q \in \mathfrak{Q}_{\lambda}$ with $L(Q)=j-n$. We apply Fubini's theorem and write

$$
\begin{aligned}
& P_{j-n+\ell(n)} T_{j}^{n} b_{Q}(x)=\int \vartheta_{n}(s) \int b_{Q}(y) \\
& \quad \times\left[\int \Phi_{j-n+\ell(n)}(x-w) K_{j}^{n}(w-y) a(s w+(1-s) y) d w\right] d y d s .
\end{aligned}
$$

Changing variables $z=w+\frac{1-s}{s} y$ we get

$$
P_{j-n+\ell(n)} T_{j}^{n} b_{Q}(x)=\int \vartheta_{n}(s) \int a(s z) \int \mathcal{A}_{j, n}^{x, z, s}(y) b_{Q}(y) d y d z d s
$$

where

$$
\mathcal{A}_{j, n}^{x, z, s}(y)=\Phi_{j-n+\ell(n)}\left(x-z+\frac{1-s}{s} y\right) K_{j}^{n}(z-y / s) .
$$

We expand $\mathcal{A}_{j, n}^{x, z, s}(y)$ about the center $y_{Q}$ of $Q$ and in view of the cancellation of $b_{Q}$ we may write

$$
\begin{aligned}
\mid P_{j-n+\ell(n)} & T_{j}^{n} b_{Q}(x) \mid \\
& \leq \iint\left|\vartheta_{n}(s) a(s z)\right|\left|\int\left(\mathcal{A}_{j, n}^{x, z, s}(y)-\mathcal{A}_{j, n}^{x, z, s}\left(y_{Q}\right)\right) b_{Q}(y) d y\right| d z d s .
\end{aligned}
$$

Using

$$
\mathcal{A}_{j, n}^{x, z, s}(y)-\mathcal{A}_{j, n}^{x, z, s}\left(y_{Q}\right)=\left\langle y-y_{Q}, \int_{0}^{1} \nabla \mathcal{A}_{j, n}^{x, z, s}\left(y_{Q}+\sigma\left(y-y_{Q}\right)\right) d \sigma\right\rangle
$$

one obtains after applying Fubini's theorem

$$
\begin{aligned}
\left\|P_{j-n+\ell(n)} T_{j}^{n} b_{Q}(x)\right\|_{1} \leq \operatorname{diam}(Q) \int_{0}^{1} \int\left|\vartheta_{n}(s)\right| \\
\times\left[\left\|\nabla \Phi_{j-n+\ell(n)}\right\|_{1} \frac{1-s}{s} \int\left|b_{Q}(y)\right| \int\left|K_{j}^{n}\left(z-\frac{y_{Q}+\sigma\left(y-y_{Q}\right)}{s}\right)\right| d z d y\right. \\
\left.\quad+\left\|\Phi_{j-n+\ell(n)}\right\|_{1} \int\left|b_{Q}(y)\right| \int \frac{1}{s}\left|\nabla K_{j}^{n}\left(z-\frac{y_{Q}+\sigma\left(y-y_{Q}\right)}{s}\right)\right| d z d y\right] d s d \sigma .
\end{aligned}
$$

Now use $\left\|\nabla K_{j}^{n}\right\|_{1} \lesssim 2^{-j+\ell_{\varepsilon}(n)}$ and $\int_{0}^{1}\left|\vartheta_{n}(s)\right| s^{-1} d s \lesssim \log n$. Since $\operatorname{diam}(Q) \lesssim 2^{j-n}$ we obtain

$$
\left\|P_{j-n+\ell(n)} T_{j}^{n} b_{Q}\right\|_{1} \lesssim \log n\left[2^{-\ell(n)}+2^{\ell_{\varepsilon}(n)-n}\right]\left\|b_{Q}\right\|_{1} \lesssim n^{-2} \log n\left\|b_{Q}\right\|_{1} .
$$

Finally we sum over all $Q \in \mathfrak{Q}_{\lambda}$ with $L(Q)=j-n$ to obtain the asserted bound. 


\section{Proof of Proposition 2.3}

Let $Q \in \mathfrak{Q}_{\lambda}$ with $L(Q)=j-n$, and let $y_{Q}$ be the center of $Q$. Fix a unit vector $\nu$, and let $\pi_{\nu}^{\perp}$ be the projection to the orthogonal complement of $\nu$, i.e. $\pi_{\nu}^{\perp}(x)=x-\langle x, \nu\rangle \nu$. In view of the support properties of the kernel it suffices to show that, for $n>n(\varepsilon)$,

$$
\left\|\left(I-P_{j-n+\ell(n)}\right)\left(I-\mathfrak{S}_{n, \nu}\right) T_{j}^{n, \nu} b_{Q}\right\|_{1} \lesssim n^{-2} 2^{-n \gamma(d-1)}\left\|b_{Q}\right\|_{1},
$$

under the additional assumption that the support of $a$ is contained in

$$
\left\{y:\left|\left\langle y-y_{Q}, \nu\right\rangle\right| \leq 2^{j+4} d,\left|\pi_{\nu}^{\perp}\left(y-y_{Q}\right)\right| \leq 2^{j+4-n \gamma} d\right\} .
$$

Note that with this hypothesis

$$
\|\widehat{a}\|_{\infty} \lesssim 2^{j d-n \gamma(d-1)}
$$

We introduce a frequency decomposition of $a$. Let $\varphi$ be a radial $C^{\infty}$ function as in $\S 2$, but now defined in $\xi$-space, so that $\varphi(\xi)=1$ for $|\xi| \leq 1$ and $\varphi(\xi)=0$ for $|\xi| \geq 6 / 5$. Define $\beta_{k}(\xi)=\varphi\left(2^{k} \xi\right)-\varphi\left(2^{k+1} \xi\right)$; then $\beta_{k}$ is supported in $\left\{\xi: 2^{-k-1} \leq|\xi| \leq \frac{6}{5} 2^{-k}\right\}$. Let $\widetilde{\beta}$ be a radial $C^{\infty}$ function so that $\widetilde{\beta}$ is supported in $\{\xi: 1 / 3 \leq|\xi| \leq 3 / 2\}$ and $\widetilde{\beta}(\xi)=1$ for $1 / 2 \leq|\xi| \leq 6 / 5$, and define $\widetilde{\beta}_{k}(\xi)=\widetilde{\beta}\left(2^{k} \xi\right)$. Then $\beta_{k} \widetilde{\beta}_{k}=\beta_{k}$. Define convolution operators $V_{k}, \Lambda_{k}$ and $\widetilde{\Lambda}_{k}$ with Fourier multipliers $\varphi\left(2^{k} \cdot\right), \beta_{k}$ and $\widetilde{\beta}_{k}$, respectively; then $\Lambda_{k} \widetilde{\Lambda}_{k}=\Lambda_{k}$ and, for every $m \in \mathbb{Z}$, the identity operator is decomposed as $I=V_{m}+\sum_{k<m} \Lambda_{k}$.

For fixed $y \in Q$ we define an operator $\mathcal{K}_{j, y}^{n, \nu}$ acting on $a$ by

$$
\mathcal{K}_{j, y}^{n, \nu}[a](x)=K_{j}^{n, \nu}(x-y) \int \vartheta_{n}(s) a(s x+(1-s) y) d s
$$

so that

$$
T_{j}^{n, \nu} b_{Q}(x)=\int b_{Q}(y) \mathcal{K}_{j, y}^{n, \nu}[a](x) d y
$$

We use dyadic frequency decompositions and split

$$
\begin{aligned}
\left(I-\mathfrak{S}_{n, \nu}\right) & \left(I-P_{j-n+\ell(n)}\right) T_{j}^{n, \nu} b_{Q} \\
& =\sum_{k_{1}} \Lambda_{k_{1}}\left(I-\mathfrak{S}_{n, \nu}\right) \widetilde{\Lambda}_{k_{1}}\left(I-P_{j-n+\ell(n)}\right) \int b_{Q}(y) \mathcal{K}_{j, y}^{n, \nu}[a] d y
\end{aligned}
$$

and then further split in (4.4)

$$
a=V_{j-n+\ell(n)} a+\sum_{k_{2}<j-n+\ell(n)} \Lambda_{k_{2}} a .
$$

We prove three lemmata with various bounds for the terms in (4.4) and (4.5). 


\section{Lemma 4.1.}

$$
\left\|\int b_{Q}(y) \mathcal{K}_{j, y}^{n, \nu}\left[V_{j-n+\ell(n)} a\right] d y\right\|_{1} \lesssim n^{-2} 2^{-n \gamma(d-1)}\left\|b_{Q}\right\|_{1} .
$$

Proof. We use the cancellation of $b_{Q}$ to estimate the left-hand side by

$$
\int\left|b_{Q}(y)\right| \int\left|\mathcal{K}_{j, y}^{n, \nu}\left[V_{j-n+\ell(n)} a\right](x)-\mathcal{K}_{j, y_{Q}}^{n, \nu}\left[V_{j-n+\ell(n)} a\right](x)\right| d x d y .
$$

For $y \in Q$ we may estimate

$$
\int\left|\mathcal{K}_{j, y}^{n, \nu}\left[V_{j-n+\ell(n)} a\right](x)-\mathcal{K}_{j, y_{Q}}^{n, \nu}\left[V_{j-n+\ell(n)} a\right](x)\right| d x \leq \mathcal{E}_{1}(y)+\mathcal{E}_{2}(y),
$$

where

$$
\mathcal{E}_{1}(y)=\left\|V_{j-n+\ell(n)} a\right\|_{\infty} \int\left|K_{j}^{n, \nu}(x-y)-K_{j}^{n, \nu}\left(x-y_{Q}\right)\right| d x
$$

and, abbreviating

$$
\begin{aligned}
& \Gamma_{j-n+\ell(n)}^{Q}(x, y, z) \\
& \quad=\int_{0}^{1}\left\langle y-y_{Q}, \nabla \mathcal{F}\left[\varphi\left(2^{j-m+\ell(n)}\right)\right]\left(s x+(1-s)\left(y_{Q}+\sigma\left(y-y_{Q}\right)\right)-z\right)\right\rangle d \sigma
\end{aligned}
$$

$\mathcal{E}_{2}$ is given by

$$
\mathcal{E}_{2}(y)=\int\left|K_{j}^{n, \nu}\left(x-y_{Q}\right)\right| \int\left|\vartheta_{n}(s)\right| \int|a(z)|\left|\Gamma_{j-n+\ell(n)}^{Q}(x, y, z)\right| d z d s d x .
$$

Now by (2.5), and since $\left|\partial_{x} \chi_{n, \nu}(x)\right| \lesssim 2^{n \gamma}|x|^{-1}$ we get

$$
\left|\mathcal{E}_{1}(y)\right| \leq\left|y-y_{Q}\right|\left\|\nabla K_{j}^{n, \nu}\right\|_{1} \lesssim 2^{j-n}\left[2^{\ell_{\varepsilon}(n)-j}+2^{n \gamma-j}\right] 2^{-n \gamma(d-1)} .
$$

Notice that for $n>n(\varepsilon)$ and $\gamma>1 / 10$ we have $2^{\ell_{\varepsilon}(n)} \lesssim 2^{n \gamma}$ and thus we see that

$$
\left|\mathcal{E}_{1}(y)\right| \lesssim 2^{-n \gamma(d-1)} n^{-2}
$$

Moreover,

$$
\begin{aligned}
\left|\mathcal{E}_{2}(y)\right| & \lesssim\left\|K_{j}^{n, \nu}\right\|_{1}\left|y-y_{Q}\right|\left\|\nabla \mathcal{F}^{-1}\left[\varphi\left(2^{j-n+\ell(n)} \cdot\right)\right]\right\|_{1} \\
& \lesssim 2^{-n \gamma(d-1)} 2^{j-n} 2^{n-j-\ell(n)} \lesssim 2^{-n \gamma(d-1)} n^{-2} .
\end{aligned}
$$

Integrating in $y$, we get

$$
\int\left(\left|\mathcal{E}_{1}(y)\right|+\left|\mathcal{E}_{2}(y)\right|\right)\left|b_{Q}(y)\right| d y \lesssim 2^{-n \gamma(d-1)} n^{-2}\left\|b_{Q}\right\|_{1},
$$

and the assertion follows. 
Lemma 4.2. Let $y \in Q$ and $a$ be as in (4.2).

(i) Let $k_{1}>k_{2}+\ell(n)+10$. Then

$$
\left\|\Lambda_{k_{1}} \mathcal{K}_{j, y}^{n, \nu}\left[\Lambda_{k_{2}} a\right]\right\|_{1} \leq C_{N} 2^{-n \gamma(d-1)} \min \left\{1, n^{2 d+2 N} 2^{n \gamma} 2^{\left(k_{2}-j+n \gamma\right) N}\right\} .
$$

(ii) Let $k_{1}<k_{2}-10$. Then

$$
\begin{aligned}
\left\|\Lambda_{k_{1}} \mathcal{K}_{j, y}^{n, \nu}\left[\Lambda_{k_{2}} a\right]\right\|_{1}+\| \Lambda_{k_{1}} & \mathcal{K}_{j, y}^{n, \nu}\left[V_{k_{2}} a\right] \|_{1} \\
& \leq C_{N} 2^{-n \gamma(d-1)} \min \left\{1,2^{n \gamma} 2^{\left(k_{1}-k_{2}\right) d} 2^{\left(k_{1}-j+n \gamma\right) N}\right\}
\end{aligned}
$$

Proof. Clearly $\left\|\mathcal{K}_{j, y}^{n, \nu}[a]\right\|_{1} \lesssim 2^{-n \gamma(d-1)}\|a\|_{\infty}$, and since the operators $\Lambda_{k}$ and $V_{k}$ are uniformly bounded we get the bound $O\left(2^{-n \gamma(d-1)}\right)$ in (i) and (ii). We seek to prove the two other bounds for $\Lambda_{k_{1}} \mathcal{K}_{j, y}^{n, \nu}\left[\Lambda_{k_{2}} a\right]$ in the two cases $k_{1}<k_{2}-10$ and $k_{1}>k_{2}+\ell(n)+10$. In (ii) the corresponding estimate for $\Lambda_{k_{1}} \mathcal{K}_{j, y}^{n, \nu}\left[V_{k_{2}} a\right]$ is entirely analogous and will be omitted.

We use the Fourier inversion formula for $a$ and for the convolution kernel of $\Lambda_{k_{1}}$; write

$$
\begin{aligned}
\Lambda_{k_{1}} \mathcal{K}_{j, y}^{n, \nu}\left[\Lambda_{k_{2}} a\right](x)= & \frac{1}{(2 \pi)^{2 d}} \int \vartheta_{n}(s) \iint \beta_{k_{1}}(\xi) \beta_{k_{2}}(\eta) \widehat{a}(\eta) \\
& \times\left[\int_{w} e^{i(\langle x-w, \xi\rangle+\langle s w+(1-s) y, \eta\rangle)} K_{j}^{n, \nu}(w-y) d w\right] d \xi d \eta d s,
\end{aligned}
$$

and integrate by parts with respect to $w$ and $\xi$. The integral can then be rewritten as ${ }^{1}$

$$
\begin{aligned}
& \frac{1}{(2 \pi)^{2 d}} \int \vartheta_{n}(s) \int \beta_{k_{2}}(\eta) \widehat{a}(\eta) \int\left[\int e^{i(\langle x-w, \xi\rangle+\langle s w+(1-s) y, \eta\rangle)}\right. \\
& \left.\times \frac{\left(I-2^{-2 k_{1}} \Delta_{\xi}\right)^{N_{1}}\left[\beta_{k_{1}}(\xi)|\xi-s \eta|^{-2 N_{2}}\right]\left(-\Delta_{w}\right)^{N_{2}} K_{j}^{n, \nu}(w-y)}{\left(1+2^{-2 k_{1}}|x-w|^{2}\right)^{N_{1}}} d w\right] d \xi d \eta d s,
\end{aligned}
$$

and we choose $N_{1}=[d / 2]+1$. Note that for $s \in \operatorname{supp}\left(\vartheta_{n}\right)$,

$$
|\xi-s \eta| \gtrsim \mathcal{C}\left(k_{1}, k_{2}, n\right):= \begin{cases}2^{-k_{2}-\ell(n)} & \text { if } k_{1}>k_{2}+\ell(n)+10 \\ 2^{-k_{1}-2} & \text { if } k_{1}<k_{2}-10 .\end{cases}
$$

Now $\left(2^{-k_{1}} \partial_{\xi}\right)^{N_{3}} \beta_{k_{1}}=O(1)$ and a computation yields

$$
\left|\left(I-2^{-2 k_{1}} \Delta_{\xi}\right)^{N_{1}}\left[\beta_{k_{1}}(\xi)|\xi-s \eta|^{-2 N_{2}}\right]\right| \lesssim\left[\mathcal{C}\left(k_{1}, k_{2}, n\right)\right]^{-N_{2}} .
$$

Moreover

$$
\begin{aligned}
\left\|\left(-\Delta_{w}\right)^{N_{2}} K_{j}^{n, \nu}\right\|_{1} & \lesssim 2^{-2 N_{2} j}\left(2^{2 N_{2} n \gamma}+2^{2 N_{2} \ell_{\varepsilon}(n)}\right) 2^{-n \gamma(d-1)} \\
& \lesssim 2^{-n \gamma(d-1)} 2^{2 N_{2}(n \gamma-j)}
\end{aligned}
$$

\footnotetext{
${ }^{1}$ Thanks to Xudong Lai who pointed out an inaccuracy in the original version of this and another formula.
} 
We integrate in $\eta$ and use that the measure of the support of $\beta_{k_{2}}$ is $O\left(2^{-k_{2} d}\right)$. Then we integrate in $x$ and $\xi$ and use that

$$
\int_{\operatorname{supp}\left(\beta_{k_{1}}\right)} \int\left(1+2^{-2 k_{1}}|x-w|^{2}\right)^{-N_{1}} d x d \xi=O(1) .
$$

Using (4.2) we then get

$$
\begin{aligned}
\| \Lambda_{k_{1}} & \mathcal{K}_{j, y}^{n, \nu}\left[\Lambda_{k_{2}} a\right]\left\|_{1} \lesssim N_{N_{2}} 2^{-k_{2} d}\right\| \widehat{a}\left\|_{\infty}\right\|(-\Delta)^{N_{2}} K_{j}^{n, \nu} \|_{1}\left[\mathcal{C}\left(k_{1}, k_{2}, n\right)\right]^{-2 N_{2}} \\
& \sum_{N_{2}} \begin{cases}2^{d \ell(n)-n \gamma(d-2)} 2^{\left(2 N_{2}-d\right)\left(k_{2}-j+\ell(n)+n \gamma\right)} & \text { if } k_{1}>k_{2}+\ell(n)+10, \\
2^{-n \gamma(d-2)} 2^{\left(2 N_{2}-d\right)\left(k_{1}-j+n \gamma\right)} 2^{\left(k_{1}-k_{2}\right) d} & \text { if } k_{1}<k_{2}-10 .\end{cases}
\end{aligned}
$$

If we put $N=2 N_{2}-d$ this gives the asserted bound for $\left\|\Lambda_{k_{1}} \mathcal{K}_{j, y}^{n, \nu}\left[\Lambda_{k_{2}} a\right]\right\|_{1}$. For $k_{1}<k_{2}-10$ the corresponding expression with $\Lambda_{k_{2}}$ replaced by $V_{k_{2}}$ is estimated in exactly the same way.

Lemma 4.3. Let $k_{2}-10 \leq k_{1} \leq k_{2}+\ell(n)+10$. Then

$$
\begin{aligned}
\| \Lambda_{k_{1}}\left(I-\mathfrak{S}^{n, \nu}\right) \mathcal{K}_{j, y}^{n, \nu}[ & \left.\Lambda_{k_{2}} a\right] \|_{1} \\
& \leq C_{N} 2^{-n \gamma(d-1)} \min \left\{1, n^{2(N+d) / \varepsilon} 2^{(d+3) n \gamma} 2^{\left(k_{1}-j+n \gamma\right) N}\right\}
\end{aligned}
$$

for every $y \in Q$.

Proof. We may again assume that (4.2) holds. Define the convolution operator $S_{n, \nu}$ by

$$
\widehat{S^{n, \nu} g}(\eta)=\phi\left(2^{n \gamma} n^{-2}\langle\nu, \eta /|\eta|\rangle\right) \widehat{g}(\eta)
$$

and split $a=S^{n, \nu} a+\left(I-S^{n, \nu}\right) a$. We shall prove the two estimates

$$
\begin{aligned}
\left\|\Lambda_{k_{1}}\left(I-\mathfrak{S}^{n, \nu}\right) \mathcal{K}_{j, y}^{n, \nu}\left[\Lambda_{k_{2}} S^{n, \nu} a\right]\right\|_{1} & \\
& \leq C_{N} n^{\left(2 \varepsilon^{-1}-4\right)(N+d)} 2^{4 n \gamma} 2^{\left(k_{1}-k_{2}\right) d} 2^{\left(k_{1}-j+n \gamma\right) N}
\end{aligned}
$$

and

$$
\left\|\Lambda_{k_{1}}\left(I-\mathfrak{S}^{n, \nu}\right) \mathcal{K}_{j, y}^{n, \nu}\left[\Lambda_{k_{2}}\left(I-S^{n, \nu}\right) a\right]\right\|_{1} \leq C_{N} n^{-5 d} 2^{4 n \gamma} 2^{\left(k_{2}-j+n \gamma\right) N} .
$$

These imply the somewhat weaker bound asserted in the lemma.

Proof of (4.6). Set

$$
b_{k_{1}, n, \nu}(\xi)=\beta_{k_{1}}(\xi)\left(1-\phi\left(2^{n \gamma} n^{-5}\langle\nu, \xi /|\xi|\rangle\right)\right)
$$

and write

$$
\begin{aligned}
(2 \pi)^{2 d} \Lambda_{k_{1}}\left(I-\mathfrak{S}^{n, \nu}\right) & \mathcal{K}_{j, y}^{n, \nu}\left[\Lambda_{k_{2}} S^{n, \nu} a\right](x) \\
=\int \vartheta_{n}( & s) \iint b_{k_{1}, n, \nu}(\xi) \beta_{k_{2}}(\eta) \phi\left(2^{n \gamma} n^{-2}\langle\nu, \eta /|\eta|\rangle\right) \widehat{a}(\eta) \\
\times & {\left[\int_{w} e^{i(\langle x-w, \xi\rangle+\langle s w+(1-s) y, \eta\rangle)} K_{j}^{n, \nu}(w-y) d w\right] d \xi d \eta d s . }
\end{aligned}
$$


If $(\xi, \eta)$ is in the support of the amplitude then for $n>10^{10}$

$$
\begin{aligned}
|\langle\xi-s \eta, \nu\rangle| & \geq|\xi||\langle\xi /|\xi|, \nu\rangle|-|\eta||\langle\eta /|\eta|, \nu\rangle| \\
& \geq|\xi|\left(2^{-n \gamma-1} n^{5}-2^{\left|k_{1}-k_{2}\right|+2} 2^{-n \gamma} n^{2}\right) \\
& \geq|\xi| 2^{-n \gamma-1}\left(n^{5}-8 \cdot 2^{\ell(n)+10} n^{2}\right) \geq 2^{-k_{1}-n \gamma} n^{5} .
\end{aligned}
$$

Now we can integrate by parts as in the proof of Lemma 4.2, except that we use the directional derivative $\left\langle\nu, \nabla_{w}\right\rangle$ instead of $\Delta_{w}$. The above integral is then estimated by

$$
\begin{aligned}
\iiint \int\left|\beta_{k_{2}}(\eta)\right||\widehat{a}(\eta)|\left|\phi\left(2^{n \gamma} n^{-2}\left\langle\nu, \frac{\eta}{|\eta|}\right\rangle\right)\right| \\
\quad \times \frac{\mid\left(I-2^{-2 k_{1}} \Delta_{\xi}\right)^{N_{1}}\left[\frac{b_{k_{1}, n, \nu}(\xi)}{\langle\xi-s \eta, \nu\rangle^{N_{2}}}\right]}{\left(1-2^{-2 k_{1}}|x-w|^{2}\right)^{N_{1}}}\left|\left\langle\nu, \nabla_{w}\right\rangle^{N_{2}} K_{j}^{n, \nu}(w-y)\right| d w d \xi d \eta d s .
\end{aligned}
$$

Observe that

$$
\left|\partial_{\xi}^{N_{3}} b_{k_{1}, n, \nu}(\xi)\right| \leq C_{N_{1}}\left(2^{n \gamma} n^{-5}\right)^{N_{3}} 2^{k_{1} N_{3}}
$$

and thus

$$
\left|\left(I-2^{-2 k_{1}} \Delta_{\xi}\right)^{N_{1}}\left[\frac{b_{k_{1}, n, \nu}(\xi)}{\langle\xi-s \eta, \nu\rangle^{N_{2}}}\right]\right| \leq C_{N_{1}}\left(2^{n \gamma} n^{-5}\right)^{2 N_{1}}\left(2^{-\left(k_{1}+n \gamma\right)} n^{5}\right)^{-N_{2}}
$$

Moreover,

$$
\left\|\left\langle\nu, \nabla_{w}\right\rangle^{N_{2}} K_{j}^{n, \nu}\right\|_{1} \leq C_{N_{2}} 2^{\left(\ell_{\varepsilon}(n)-j\right) N_{2}} 2^{-n \gamma(d-1)} .
$$

We assume $2 N_{1}>d$, integrate in $x$ and $\xi$, and use (4.8). Then we obtain

$$
\begin{aligned}
\| \Lambda_{k_{1}}\left(I-\mathfrak{S}^{n, \nu}\right) \mathcal{K}_{j, y}^{n, \nu} & {\left[\Lambda_{k_{2}} S^{n, \nu} a\right] \|_{1} } \\
& \lesssim_{N_{1}, N_{2}}\left(2^{2 n \gamma} n^{-5}\right)^{2 N_{1}}\|\widehat{a}\|_{\infty} 2^{-k_{2} d} \frac{2^{\left(\ell_{\varepsilon}(n)-j\right) N_{2}} 2^{-n \gamma(d-1)}}{\left(2^{-k_{1}-n \gamma} n^{5}\right)^{N_{2}}} .
\end{aligned}
$$

We use (4.2) and that the support of $\eta \mapsto \beta_{k_{2}}(\eta)$ has measure $O\left(2^{-k_{2} d}\right)$. Thus the expression in the previous displayed inequality can be crudely estimated by

$$
C_{N_{1}, N_{2}} n^{\left(2 \varepsilon^{-1}-4\right) N_{2}-10 N_{1}} 2^{n \gamma\left(2 N_{1}-d+2\right)} 2^{\left(k_{1}-k_{2}\right) d} 2^{\left(k_{1}-j+n \gamma\right)\left(N_{2}-d\right)}
$$

and, if we chose the integer $N_{1} \in\{(d+1) / 2,(d+2) / 2\}$ and $N=N_{2}-d$, we obtain (4.6).

Proof of (4.7). Set

$$
\widetilde{b}_{k_{2}, n, \nu}(\eta)=\beta_{k_{2}}(\eta)\left(1-\phi\left(2^{n \gamma} n^{-2}\langle\nu, \eta /|\eta|\rangle\right)\right)
$$

and write

$$
\begin{aligned}
(2 \pi)^{d} \Lambda_{k_{1}}\left(I-\mathfrak{S}^{n, \nu}\right) \mathcal{K}_{j, y}^{n, \nu}\left[\Lambda_{k_{2}}\left(I-S^{n, \nu}\right) a\right](x) & \\
=\int K_{j}^{n, \nu}(w-y) \iint b_{k_{1}, n, \nu}(\xi) \widetilde{b}_{k_{2}, n, \nu}(\eta) \widehat{a}(\eta) & \\
\times & {\left[\int \vartheta_{n}(s) e^{i(\langle x-w, \xi\rangle+\langle s w+(1-s) y, \eta\rangle)} d s\right] d \xi d \eta d w . }
\end{aligned}
$$


Now if $w-y \in \operatorname{supp}\left(K_{j}^{n, \nu}\right)$ then $\left|\frac{w-y}{|w-y|}-\nu\right| \leq 2^{-n \gamma}$ and if $\eta \in \operatorname{supp}\left(\widetilde{b}_{k_{2}, n, \nu}\right)$ we get

$$
|\langle w-y, \eta\rangle| \geq|w-y|\left(\langle\nu, \eta\rangle-|\eta| 2^{-n \gamma}\right) \geq|w-y||\eta| 2^{-n \gamma}\left(\frac{1}{2} n^{2}-1\right)
$$

and hence

$$
|\langle w-y, \eta\rangle| \geq 2^{j-k_{2}-n \gamma-4} n^{2}
$$

Integration by parts with respect to $s$ yields

$$
\begin{aligned}
& (2 \pi)^{d} \Lambda_{k_{1}}\left(I-\mathfrak{S}^{n, \nu}\right) \mathcal{K}_{j, y}^{n, \nu}\left[\Lambda_{k_{2}}\left(I-S^{n, \nu}\right) a\right](x)= \\
& \int K_{j}^{n, \nu}(w-y) \iint \widehat{a}(\eta) \widetilde{b}_{k_{2}, n, \nu}(\eta) \frac{\left(I-2^{-2 k_{1}} \Delta_{\xi}\right)^{N_{1}} b_{k_{1}, n, \nu}(\xi)}{\left(1+2^{-2 k_{1}}|x-w|^{2}\right)^{N_{1}}} e^{i(\langle x-w, \xi\rangle+\langle y, \eta\rangle)} \\
& \times\left[\int \vartheta_{n}^{\left(N_{3}\right)}(s) \frac{i^{N_{3}} e^{i s\langle w-y, \eta\rangle}}{\langle w-y, \eta\rangle^{N_{3}}} d s\right] d \xi d \eta d w
\end{aligned}
$$

We apply this with $N_{1}>d / 2$ and, using (4.2), (4.9), and (4.10), obtain

$$
\begin{aligned}
\| \Lambda_{k_{1}} & \left(I-\mathfrak{S}^{n, \nu}\right) \mathcal{K}_{j, y}^{n, \nu} \Lambda_{k_{2}}\left(I-S^{n, \nu}\right) a \|_{1} \\
& \lesssim N_{1}, N_{3}\left(2^{n \gamma} n^{-5}\right)^{2 N_{1}}\left\|K_{j}^{n, \nu}\right\|_{1} \frac{\left\|\vartheta_{n}^{\left(N_{3}\right)}\right\|_{1}}{\left(2^{j-k_{2}-n \gamma-4} n^{2}\right)^{N_{3}}} 2^{-k_{2} d}\|\widehat{a}\|_{\infty} \\
& \lesssim N_{1}, N_{3} n^{-2-10 N_{1}} 2^{n \gamma\left(2 N_{1}-d+2\right)} 2^{\left(k_{2}-j+n \gamma\right)\left(N_{3}-d\right)} .
\end{aligned}
$$

Inequality (4.7) follows if we choose $N=N_{3}-d$ and $N_{1} \in\{(d+1) / 2,(d+2) / 2\}$.

Proof of Proposition 2.3, conclusion. Let, for fixed $n, \nu$ and $j$, and for a fixed cube $Q \in \mathfrak{Q}_{\lambda}$ with $L(Q)=j-n$,

$$
I_{k_{1}}=\widetilde{\Lambda}_{k_{1}}\left(I-P_{j-n+\ell(n)}\right) \Lambda_{k_{1}}\left(I-\mathfrak{S}_{n, \nu}\right)\left[\int b_{Q}(y) \mathcal{K}_{j, y}^{n, \nu}\left[V_{j-n+\ell(n)} a\right] d y\right]
$$

and

$$
I I_{k_{1}, k_{2}}=\widetilde{\Lambda}_{k_{1}}\left(I-P_{j-n+\ell(n)}\right) \Lambda_{k_{1}}\left(I-\mathfrak{S}_{n, \nu}\right)\left[\int b_{Q}(y) \mathcal{K}_{j, y}^{n, \nu}\left[\Lambda_{k_{2}} a\right](x) d y\right] .
$$

By (4.4) and (4.5) it is enough to show that

$$
\sum_{k_{1}}\left\|I_{k_{1}}\right\|_{1}+\sum_{k_{1}} \sum_{k_{2}<j-n+\ell(n)}\left\|I I_{k_{1}, k_{2}}\right\|_{1} \lesssim n^{-2} 2^{-\gamma n(d-1)}\left\|b_{Q}\right\|_{1} .
$$

We have

$$
\left\|\Lambda_{k_{1}}\left(I-\mathfrak{S}_{n, \nu}\right)\right\|_{L^{1} \rightarrow L^{1}} \leq C
$$


uniformly in $n, \nu$ and $k_{1}$, and using the support and cancellation properties of the kernel of $I-P_{j-n+\ell(n)}$ we also have

$$
\left\|\widetilde{\Lambda}_{k_{1}}\left(I-P_{j-n+\ell(n)}\right)\right\|_{L^{1} \rightarrow L^{1}} \lesssim \min \left\{1,2^{j-n+\ell(n)-k_{1}}\right\} .
$$

Lemma 4.1 together with (4.13) and (4.12) immediately gives

$$
\sum_{k_{1} \geq j-n+\ell(n)-10}\left\|I_{k_{1}}\right\|_{1} \lesssim n^{-2} 2^{-\gamma n(d-1)}\left\|b_{Q}\right\|_{1} .
$$

It remains to verify that the other terms satisfy better bounds, namely

$$
\begin{aligned}
\sum_{k_{1}<j-n+\ell(n)-10}\left\|I_{k_{1}}\right\|_{1}+\sum_{k_{1}} \sum_{k_{2}<j-n+\ell(n)}\left\|I I_{k_{1}, k_{2}}\right\|_{1} \\
\quad \lesssim C_{N} n^{A_{1} N} 2^{A_{2} n} 2^{n(\gamma-1) N}\left\|b_{Q}\right\|_{1}
\end{aligned}
$$

for all $N$, and suitable $A_{1} \leq 10 d / \varepsilon$ and $A_{2} \leq 10$. Choose $N=100 d$. Taking into account that $\gamma \leq 9 / 10$ one can check that the bound in (4.15) is dominated by $C n^{-2} 2^{-n \gamma(d-1)}\left\|b_{Q}\right\|_{1}$ for all $n$ with $n^{-1} \log n \leq 10^{-4} \varepsilon / d$, which is satisfied for $n>n(\varepsilon)$.

For the terms involving $I_{k_{1}}$, with $k_{1} \geq j-n+\ell(n)+10$ we get by the second estimate in part (ii) of Lemma 4.2 , with $k_{2}=j-n+\ell(n)$,

$$
\begin{aligned}
& \sum_{k_{1}<j-n+\ell(n)-10}\left\|I_{k_{1}}\right\|_{1} \\
& \lesssim_{N} 2^{-n \gamma(d-2)} \sum_{k_{1}<j-n+\ell(n)-10} 2^{\left(k_{1}-j+n-\ell(n)\right) d} 2^{\left(k_{1}-j+n \gamma\right) N}\left\|b_{Q}\right\|_{1} \\
& \lesssim_{N} 2^{-n \gamma(d-2)}\left(2^{n(\gamma-1)} n^{2}\right)^{N}\left\|b_{Q}\right\|_{1} .
\end{aligned}
$$

Next consider $\sum_{k_{1}, k_{2}}\left\|I I_{k_{1}, k_{2}}\right\|_{1}$ where the $k_{2}$-summation is extended over $k_{2}<$ $j-n+\ell(n)$. For $k_{1} \geq j-n+\ell-10$ we can sum a geometric series in $k_{1}$, with a uniform bound, due to (4.13). By Lemma 4.2, part (i)

$$
\begin{aligned}
& \sum_{\substack{k_{1} \geq j-n+\ell(n)-10 \\
\left(k_{1}, k_{2}\right)::_{k_{2}<\min \left\{k_{1}-\ell(n)-10, j-n+\ell(n)\right\}}}}\left\|I I_{k_{1}, k_{2}}\right\|_{1} \\
& \lesssim 2^{-n \gamma(d-2)} n^{2 d+2 N} \sum_{k_{2}<j-n+\ell(n)} 2^{\left(k_{2}-j+n \gamma\right) N}\left\|b_{Q}\right\|_{1} \\
& \lesssim 2^{-n \gamma(d-2)} n^{2 d+4 N} 2^{n(\gamma-1) N}\left\|b_{Q}\right\|_{1},
\end{aligned}
$$

and by Lemma 4.3

$$
\begin{aligned}
\sum_{\substack{k_{1} \geq j-n+\ell(n)-10 \\
k_{1}-\ell(n)-10 \leq k_{2} \leq k_{1}+10 \\
k_{2}<j-n+\ell(n)}}\left\|I I_{k_{1}, k_{2}}\right\|_{1} & \lesssim\left\|b_{Q}\right\|_{1} \ell(n) n^{2(N+d) / \varepsilon} 2^{4 n \gamma} \sum_{k_{1} \leq j-n+2 \ell(n)+10} 2^{\left(k_{1}-j+n \gamma\right) N} \\
& \lesssim\left\|b_{Q}\right\|_{1} \log (n) n^{2(N+d)\left(\varepsilon^{-1}+2\right)} 2^{n(\gamma-1) N} .
\end{aligned}
$$


The case $k_{2}>k_{1}+10$ does not occur when $k_{1} \geq j-n+\ell(n)-10$ because of the restriction $k_{2}<j-n+\ell(n)$. Thus in all cases of (4.15) which involve the restriction $k_{1} \geq j-n+\ell(n)-10$ we obtain the required estimate.

Now sum the terms $\left\|I I_{k_{1}, k_{2}}\right\|_{1}$ with $k_{1}<j-n+\ell(n)-10$. By Lemma 4.2, part (i),

$$
\begin{aligned}
\sum_{\substack{\left(k_{1}, k_{2}\right): k_{1}<j-n+\ell(n)-10 \\
k_{2}<k_{1}-\ell(n)-10} I I_{k_{1}, k_{2}} \|_{1}} & \lesssim n^{2 d+2 N} 2^{-n \gamma(d-2)} \sum_{\left(k_{1}, k_{2}\right):} \sum_{\substack{k_{1}<j-n+\ell(n)-10 \\
k_{2}<k_{1}-\ell(n)-10}} 2^{\left(k_{2}-j+n \gamma\right) N}\left\|b_{Q}\right\|_{1} \\
& \lesssim n^{2 d+2 N} 2^{-n \gamma(d-2)} 2^{n(\gamma-1) N}\left\|b_{Q}\right\|_{1} ;
\end{aligned}
$$

by Lemma 4.2 , part (ii),

$$
\begin{aligned}
& \sum_{\substack{k_{1}<j-n+\ell(n)-10 \\
\left(k_{1}, k_{2}\right): k_{1}+10<k_{2}<j-n+\ell(n)-10}}\left\|I I_{k_{1}, k_{2}}\right\|_{1} \\
& \quad \lesssim 2^{-n \gamma(d-2)} \sum_{k_{1}<j-n+\ell(n)-10} 2^{\left(k_{1}-j+n \gamma\right) N} \sum_{k_{2}>k_{1}+10} 2^{\left(k_{1}-k_{2}\right) d}\left\|b_{Q}\right\|_{1}, \\
& \lesssim n^{2 N} 2^{-n \gamma(d-2)} 2^{n(\gamma-1) N}\left\|b_{Q}\right\|_{1},
\end{aligned}
$$

and finally, by Lemma 4.3,

$$
\begin{aligned}
\sum_{\substack{k_{1}<j-n+\ell(n)-10 \\
\left(k_{1}, k_{2}\right):}}\left\|I I_{k_{1}, k_{2}}\right\|_{1} & \lesssim \log (n) n^{2(N+d) / \varepsilon} 2^{4 n \gamma} \sum_{k_{1} \leq j-n+\ell(n)} 2^{\left(k_{1}-j+n \gamma\right) N}\left\|b_{Q}\right\|_{1} \\
& \lesssim n^{2(N+d)\left(\varepsilon^{-1}+1\right)} 2^{4 n \gamma} 2^{n(\gamma-1) N}\left\|b_{Q}\right\|_{1} .
\end{aligned}
$$

This finishes the proof of (4.15).

\section{Proof of Proposition 2.4}

We use a slightly modified version of an argument in [8]. The main observation is that, for fixed $n>0$, we have

$$
\sup _{\xi \neq 0} \sum_{\nu \in \Theta_{n}}\left|\phi\left(2^{n \gamma} n^{-5}\langle\nu, \xi /|\xi|\rangle\right)\right| \lesssim 2^{n \gamma(d-2)} n^{5} .
$$

To see this it suffices, by homogeneity, to take the supremum over all $\xi \in S^{d-1}$. Now if $|\xi|=1$ and $\phi\left(2^{n \gamma} n^{-5}\langle\theta, \xi\rangle\right) \neq 0$ then the distance of $\nu$ to the hyperplane $\xi$ is at most $C n^{5} 2^{-n \gamma}$ and since the vectors in $\Theta_{n}$ are $c 2^{-n \gamma}$-separated there are $O\left(2^{n \gamma(d-2)} n^{5}\right)$ such vectors, hence (5.1) holds.

From (5.1) it follows that

$$
\begin{aligned}
\left\|\sum_{\nu \in \Theta_{n}} \mathfrak{S}_{n, \nu} \sum_{j}\left(I-P_{j-n+\ell(n)}\right) T_{j}^{n, \nu} B_{j-n}\right\|_{2}^{2} & \\
& \lesssim 2^{n \gamma(d-2)} n^{5} \sum_{\nu \in \Theta_{n}}\left\|\sum_{j}\left(I-P_{j-n+\ell(n)}\right) T_{j}^{n, \nu} B_{j-n}\right\|_{2}^{2}
\end{aligned}
$$


and since $\# \Theta_{n} \lesssim 2^{n \gamma(d-1)}$ the asserted inequality is a consequence of

$$
\left\|\sum_{j}\left(I-P_{j-n+\ell(n)}\right) T_{j}^{n, \nu} B_{j-n}\right\|_{2}^{2} \lesssim 2^{-2 n \gamma(d-1)} \lambda\|f\|_{1}
$$

for each $\nu \in \Theta_{n}$.

For the proof of (5.2) the cancellation of $B_{j-n}$ plays no role. Let

$$
H_{j}^{n, \nu}(x)=2^{-j d} \chi_{\tau_{j}^{n, \nu}}(x) .
$$

where

$$
\tau_{j}^{n, \nu}=\left\{x:|\langle x, \nu\rangle| \leq 2^{j+2},|x-\langle x, \nu\rangle| \leq 2^{j+2-\gamma n}\right\} .
$$

Then from (1.1) we get

$$
\left|\left(I-P_{j-n+\ell(n)}\right) T_{j}^{n, \nu} B_{j-n}(x)\right| \lesssim H_{j}^{n, \nu} *\left|B_{j-n}\right|(x) .
$$

Therefore

$$
\begin{aligned}
& \left\|\sum_{j}\left(I-P_{j-n+\ell(n)}\right) T_{j}^{n, \nu} B_{j-n}\right\|_{2}^{2} \\
& \quad \lesssim 2 \sum_{j} \int\left|B_{j-n}(x)\right| \sum_{i \leq j} H_{j}^{n, \nu} * H_{i}^{n, \nu} *\left|B_{i-n}(x)\right| d x .
\end{aligned}
$$

Observe that $\left\|H_{i}^{n, \nu}\right\|_{1} \lesssim 2^{-i d} \operatorname{meas}\left(\tau_{i}^{n, \nu}\right) \lesssim 2^{-n \gamma(d-1)}$ and thus

$$
H_{j}^{n, \nu} * H_{i}^{n, \nu}(x) \lesssim 2^{-n \gamma(d-1)} 2^{-j d} \chi_{\widetilde{\tau}_{j}^{n, \nu}}(x)
$$

where $\widetilde{\tau}_{j}^{n, \nu}$ is the double of $\tau_{j}^{n, \nu}$. Hence, for each $x \in \mathbb{R}^{d}$ and $j \in \mathbb{Z}$,

$$
\begin{aligned}
\sum_{i \leq j} H_{j}^{n, \nu} * H_{i}^{n, \nu} *\left|B_{i-n}\right|(x) & \lesssim 2^{-n \gamma(d-1)} 2^{-j d} \sum_{i \leq j} \int_{x+\widetilde{\tau}_{j}^{n, \nu}}\left|B_{i-n}(y)\right| d y \\
& \lesssim 2^{-n \gamma(d-1)} 2^{-j d} \sum_{i \leq j} \sum_{\substack{Q \in \mathfrak{Q}_{\lambda}: \\
L(Q)=i-n \\
Q \cap\left(x+\widetilde{\tau}_{j}^{n, \nu}\right) \neq \emptyset}} \int\left|b_{Q}(x)\right| d x \\
& \lesssim 2^{-n \gamma(d-1)} 2^{-j d} \lambda \operatorname{meas}\left(\widetilde{\tau}_{j}^{n, \nu}\right) \lesssim 2^{-2 n \gamma(d-1)} \lambda
\end{aligned}
$$

Here we have used $\left\|b_{Q}\right\|_{1} \lesssim \lambda|Q|$, and the disjointness of the interiors of the cubes $Q$ in $\mathfrak{Q}_{\lambda}$. Thus we get the estimate

$$
\left\|\sum_{j}\left(I-P_{j-n+\ell(n)}\right) T_{j}^{n, \nu} B_{j-n}\right\|_{2}^{2} \lesssim 2^{-2 n \gamma(d-1)} \lambda \sum_{j}\left\|B_{j-n}\right\|_{1},
$$

which yields (5.2). 


\section{Open problems}

\subsection{Principal value integrals}

Let

$$
\mathcal{T}_{r} f(x)=\int_{|x-y|>r} K(x-y) \int_{0}^{1} a(s x+(1-s) y) d s f(y) d y .
$$

Our proof shows that the operators $\mathcal{T}_{r}$ are of weak type $(1,1)$, with uniform bounds; moreover, for $f \in L^{1}, \mathcal{T}_{r} f$ converges in measure to $T f$ where $T$ is weak type $(1,1)$. However it is currently open whether the principal value $\lim _{r \rightarrow 0} \mathcal{T}_{r} f(x)$ exists for almost every $x \in \mathbb{R}^{d}$. By Stein's theorem [9] this is equivalent to the open question whether the maximal singular integral $\sup _{r>0}\left|\mathcal{T}_{r} f\right|$ defines an operator of weak type $(1,1)$.

\subsection{Principal value integrals for rough singular convolution operators}

The question analogous to 6.1 is open for classical singular integral operators with rough convolution kernel $\Omega(y /|y|)|y|^{-d}$ where $\Omega \in L \log L\left(S^{d-1}\right), d \geq 2$ and $\int_{S^{d-1}} \Omega(\theta) d \sigma=0$. These operators are known to be of weak type $(1,1),[8]$, but the a.e. existence of the principal value integrals is open even for $\Omega \in L^{\infty}\left(S^{d-1}\right)$.

\subsection{Christ-Journé operators}

Let $F \in C^{\infty}(\mathbb{R})$, let $K$ be a Calderón-Zygmund convolution kernel, and let $a \in$ $L^{\infty}\left(\mathbb{R}^{d}\right)$. Christ and Journé [3] showed that the operator defined for $f \in C_{0}^{\infty}\left(\mathbb{R}^{d}\right)$ by

$$
\mathcal{T} f(x)=p . v . \int F\left(\int_{0}^{1} a(s x+(1-s) y) d t\right) K(x-y) f(y) d y
$$

extends to a bounded operator on $L^{p}\left(\mathbb{R}^{d}\right), 1<p<\infty$. It would be interesting to get the weak type $(1,1)$ inequality for nonlinear $F$, in dimension $d \geq 2$.

\section{References}

[1] Calderón, A. P.: Commutators of singular integral operators. Proc. Nat. Acad. Sci. USA 53 (1965), 1092-1099.

[2] Christ, M.: Weak type $(1,1)$ bounds for rough operators. Annals of Math. 128 (1988), no. 1, 19-42.

[3] Christ, M. And Journé, J.-L.: Polynomial growth estimates for multilinear singular integral operators. Acta Math. 159 (1987), no. 1-2, 51-80.

[4] Christ, M. and Rubio de Francia, J.-L.: Weak type $(1,1)$ bounds for rough operators, II. Invent. Math. 93 (1988), no. 1, 225-237.

[5] Fefferman, C.: Inequalities for strongly singular convolution operators. Acta Math. 124 (1970), 9-36.

[6] Grafakos, L. And Honzík, P.: A weak type estimate for commutators. Int. Math. Res. Not. IMRN 2012, no. 20, 4785-4796. 
[7] Hofmann, S.: Weak $(1,1)$ boundedness of singular integrals with nonsmooth kernel. Proc. Amer. Math. Soc. 103 (1988), no. 1, 260-264.

[8] Seeger, A.: Singular integral operators with rough convolution kernels. J. Amer. Math. Soc. 9 (1996), no. 1, 95-105.

[9] Stein, E. M.: On limits of seqences of operators. Annals of Math. (2) 74 (1961), 140-170.

[10] Stein, E. M.: Singular integrals and differentiability properties of functions. Princeton University Press, Princeton, NJ, 1970.

[11] TAO, T.: The weak-type $(1,1)$ of $L \log L$ homogeneous convolution operator. Indiana Univ. Math. J. 48 (1999), no. 4, 1547-1584.

Received September 20, 2012.

Andreas Seeger: Department of Mathematics, University of Wisconsin-Madison, Madison, WI 53706, USA.

E-mail: seeger@math.wisc.edu 